\title{
EI MERCOSUR a 20 años del Protocolo de Ouro Preto: un balance de la dimensión comercial
}

\section{MERCOSUR at 20 years of the "Protocol of Ouro Preto": a balance of its commercial dimension}

Julieta Zelicovich*

\section{Resumen}

Este artículo realiza un análisis de la dimensión comercial del MERCOSUR. Para ello se distinguen dos niveles: el de la política comercial implementada por los países miembros en sus relaciones hacia el bloque; y el de los flujos del comercio. A 20 años del Protocolo de Ouro Preto, se argumenta que el MERCOSUR, si bien no ha alcanzado los objetivos de un mercado común, ha logrado cimentar las bases de una interdependencia económica. Se observa que en los últimos diez años, a pesar de que la coordinación de políticas hacia la zona de libre comercio y unión aduanera ha sido endeble, los flujos del comercio intrazona han mantenido una relativa estabilidad. El análisis combina elementos cuantitativos y cualitativos en su diseño de investigación y se apoya en fuentes documentales y estadísticas, que permiten presentar argumentos y evidencia empírica que contrastan con las interpretaciones pesimistas del devenir del bloque regional.

Palabras clave: Mercosur; integración regional; Ouro Preto; política comercial; comercio

Doctora en Relaciones Internacionales, Universidad Nacional de Rosario, Argentina. Becaria Pos Doctoral del Consejo Nacional de Investigaciones Científicas y Técnicas (CONICET), Argentina.

Contacto: julieta.zelicovich@fcpolit.unr.edu.ar 


\begin{abstract}
This paper aims to analyse MERCOSUR's commercial dimension. For that, it distinguishes two levels: the one of the trade policy implemented by the country members towards the regional block, and the one of the trade flows among MECOSUR. 20 years after the Protocol of Ouro Preto, we argue that although MERCOSUR has not achieved the targets of a common market, it has managed to lay the foundations of an economic interdependence. In the last ten years, even though the coordination of the politics towards the free trade area and the custom union have been weak, the trade flows have maintained a relative stability. The research design combines quantitative and qualitative elements, and it is based in documental and statistic's resources, that allow to present arguments and empirical evidence that contrast against the pessimistic interpretations of the future of the regional bloc.
\end{abstract}

Key words: MERCOSUR; Regional Integration; Ouro Preto; comercial policy; trade.

\title{
Clasificación/Classification JEL: F150, F130, F59
}

\section{Introducción}

El estado de la integración regional en América Latina es un tema de análisis frecuente en la disciplina de las relaciones internacionales. Desde hace algunos años, en estas recurrentes revisitas al tema, por un lado, se ha extendido una interpretación que enfatiza que los procesos de integración en la región se encuentran estancados o en crisis, especialmente en cuanto al cumplimiento de sus objetivos de establecer uniones aduaneras o zonas de libre comercio ${ }^{1}$. Por otra parte, se ha acentuado la difusión de análisis de los procesos de integración desde la perspectiva del regionalismo "post liberal"2. Como resultado, han primado visiones pesimistas respecto del devenir de bloques regionales como el MERCOSUR; a la vez que se han hecho menos recurrentes los estudios que se centran en la dimensión comercial de la integración.

En ese contexto, este artículo tiene por objetivo realizar un análisis sobre la situación de la dimensión comercial del MERCOSUR, a 20 años de la firma del Protocolo de Ouro Preto. Para ello se propone distinguir dos niveles de análisis: uno el de la política comercial externa, y otro,

1 Debe señalarse que el MERCOSUR encabeza el listado de los casos que se suele considerar dentro de los análisis que toman esta perspectiva. Sin embargo, estas interpretaciones han priorizado las rupturas por sobre las continuidades, así como las dificultades de avance de algunos grandes temas, en vez de la progresión de otros de menor envergadura. Véase Bouzas, Motta Veiga y Ríos (2007) y Cienfuegos y Sanahuja (2010), entre otros.

2 Por ejemplo, Serbin, Martínez y Ramanzini (2012). 
el de los flujos del comercio. El primero se refiere a las políticas, acciones e instrumentos de la unión aduanera, que cada uno de los Estados Partes del MERCOSUR aplica respecto de ese comercio, y en particular al grado de cooperación existente en ello. El segundo remite a los intercambios de bienes que se realizan entre los países miembros del bloque.

El argumento que se sostiene es que, a lo largo de la última década, mientras que desde la perspectiva de la política comercial el MERCOSUR ha presentado un bajo nivel de coordinación respecto de la unión aduanera; los flujos del comercio se han mantenido relativamente estables; ello, a pesar de las diferencias observadas en la dimensión de coordinación política.

De esta forma, el artículo presenta argumentos y evidencia empírica que contrasta con las lecturas más críticas respecto del estado actual del MERCOSUR. Si bien es cierto que los objetivos del establecimiento de un mercado común están lejos de haberse alcanzado, no puede afirmarse que en materia de relaciones económicas y comerciales el bloque no haya alcanzado una notable interdependencia entre sus países miembros. En efecto, existe una dinámica económica que es preciso reconocer.

El análisis combina elementos cuantitativos y cualitativos en su diseño de investigación, y se apoya en fuentes documentales y estadísticas. Respecto de estas últimas, es preciso notar que, al no disponerse de datos sistematizados y comparables del flujo de comercio de Venezuela en el MERCOSUR, en todos los casos el estudio refiere a los cuatro miembros originarios del bloque: Argentina, Brasil, Uruguay y Paraguay. En la segunda sección se presenta de forma abreviada la evolución del MERCOSUR desde su creación a la actualidad. Seguidamente, se analizan las relaciones de política comercial de los miembros del bloque, enfatizando especialmente el grado de cooperación existente en tres planos: el de la zona de libre comercio, el de la implementación de la unión aduanera y el de las estrategias de negociaciones comerciales externas. En un cuarto apartado se detalla la evolución de los flujos del comercio. Allíse consideran el volumen, la intensidad y la composición del intercambio de bienes. Finalmente, se exponen las conclusiones del análisis.

\section{2. ¿El comercio impulsa a la política, o viceversa? Algunos apuntes sobre esta relación en el origen del MERCOSUR}

Los antecedentes del MERCOSUR fueron más bien políticos, antes que económicos y comerciales. A mediados de los años 80, con el regreso de las democracias a la región, los 
presidentes Raúl Alfonsín y José Sarney se propusieron buscar un mecanismo que terminara con las rivalidades entre Argentina y Brasil, y con la concepción del otro como amenaza, fortaleciendo la paz regional. Una de las vías para lograrlo era la integración regional. El razonamiento que ambos Presidentes esgrimían señalaba que con la democracia era posible avanzar en proyectos comunes en materia de desarrollo industrial, infraestructura energética y cooperación científica; y que dichos emprendimientos cimentarían intereses conjuntos que reasegurarían la paz regional y posibilitarían la inserción de ambos países en el mundo (Alfonsín, 1987; Flores, 2004).

En términos comerciales, el modelo regional era el de la Asociación Latinoamericana de Integración (ALADI): un modelo de alta flexibilidad, basado en la celebración de acuerdos de alcance parcial y acuerdos de complementación económica entre las partes que eventualmente convergerían entre sí. Dentro de la Asociación, estos últimos acuerdos -los ACE- sostenían como objetivos "promover el máximo aprovechamiento de los factores de la producción, estimular la complementación económica, asegurar condiciones equitativas de competencia, facilitar la concurrencia de los productos al mercado internacional e impulsar el desarrollo equilibrado y armónico de los países miembros", un esquema similar al seguido por Argentina, Brasil, Paraguay y Uruguay en su camino hacia el MERCOSUR.

En efecto, en noviembre de 1985 se firmó la Declaración de Iguazú, y en 1986 Argentina y Brasil suscribieron el Acta de Cooperación e Integración Argentino-Brasileña, así como el Programa de Intercambio y Cooperación Económica (PICE), el cual avanzaba en un enfoque sectorial, aunque concentrado en áreas poco sensibles, donde no existía producción doméstica o en los que operaban empresas transnacionales que ya habían ensayado esquemas de división del trabajo (Bouzas y Pagnota, 2003: 43) ${ }^{3}$. Dos años más tarde se firmó el Tratado de Cooperación y Desarrollo, en el cual "por primera vez se institucionalizaba la idea de crear un espacio económico común y generalizado, mediante la remoción de los obstáculos tarifarios y no tarifarios al comercio de bienes y servicios" (Hilliar, 1996: 9).

En los años 90, con la consolidación del pensamiento neoliberal entre los gobiernos de la región y la hegemonía del paradigma del regionalismo abierto, se firmaron el Acta de Buenos Aires (1990), el Tratado de Asunción (1991) y el Protocolo de Ouro Preto (1994). En estos documentos se sentaron las bases de la política comercial del MERCOSUR.

3 "Durante este período de poco más de dos años se generaron 24 Protocolos, agrupables bajo cuatro rubros posibles: a) comerciales, b) científico-tecnológicos, c) de infraestructura y d) de articulación estructural." (Hilliar, 1996). 
El Tratado de Asunción estableció los mecanismos para la constitución de un espacio común a través de la implementación de un cronograma de desgravaciones arancelarias que llegaba al 100\% en un período de cuatro años, que debería ser ampliado luego a la categoría de mercado común -objetivo principal del tratado. Complementariamente, el Protocolo de Ouro Preto estableció la estructura institucional del bloque y la subjetividad internacional del MERCOSUR, a la vez que avanzó hacia la creación de una unión aduanera con la aplicación de un arancel externo común. El enfoque sectorial y gradual del PICE fue reemplazado por uno lineal y, para muchos, apresurado o irrealista (Caetano, 2011).

En poco tiempo se generó un espacio de integración de manera acelerada aunque incompleta: elementos constitutivos de un único territorio aduanero como el que se pretendía alcanzar no fueron incorporados al proceso. Entre otros, la armonización de las normativas internas vinculadas al comercio exterior y a la radicación de inversiones, la efectiva coordinación de la política macroeconómica, o bien la reducción de las asimetrías, fueron abordadas de manera acotada y/o tardíamente. Por ejemplo, no existieron mecanismos de coordinación antelas devaluaciones de las monedas; el Fondo para la Convergencia Estructural del MERCOSUR (FOCEM) se volvió operativo en 2006; y recién en 2010 el bloque se dio su propio Código Aduanero -en proceso de ratificación-. La implementación del arancel externo común tuvo cuantiosas excepciones; no se lograron coordinar mecanismos de redistribución de la renta aduanera, ni respecto del doble cobro del arancel, sino hasta 2010, quedando aún pendiente su plena puesta en vigencia ${ }^{4}$. Asimismo, persistieron divergencias a raíz de las asimetrías en la internalización de las normas y en la jerarquía constitucional del MERCOSUR al interior de cada uno de los países.

Aun con dichos temas pendientes, el MERCOSUR prontamente generó un efecto político y económico entre sus países miembros. En particular, en términos de comercio, "desde 1985 y hasta 1997 la tasa de crecimiento anual del comercio intrarregional fue entre un 15\% y un $27 \%$ anual, quintuplicando la del comercio extrazona” (Rapoport, 2010:427). El comercio intrazona pasó de ser un 8\% a comienzos de los años 90, al 23\%, en su valor máximo, en 1998. Con la crisis de la devaluación del Real en 1998 y la crisis argentina de 2001, el comercio intrazona se redujo; y de hecho, cada vez que las economías mayores atravesaron periodos recesivos, la oposición doméstica de los cuatro países hacia el MERCOSUR se incrementó,

4 Véase Caetano (2011). En 2010 se aprobó la Decisión 10/10 del Consejo del Mercado Común, a través de la cual se aprobaron los "Lineamientos para la implementación de la eliminación del doble cobro del AEC y Distribución de la renta aduanera". Se previó comenzar la puesta en vigor de lo establecido en diferentes etapas, entre 2012 y 2019. 
aunque sin eco entre los gobiernos. La zona se constituyó en un espacio de paz (objetivo primario del gobierno de Alfonsín), y el MERCOSUR en un elemento constitutivo de las políticas exteriores de los gobiernos. Con los años, algunos países de la región se convirtieron en miembros asociados (Chile, Bolivia ${ }^{5}$, Perú, Ecuador y Colombia) y otros en miembros plenos (Venezuela, aunque en período de transición).

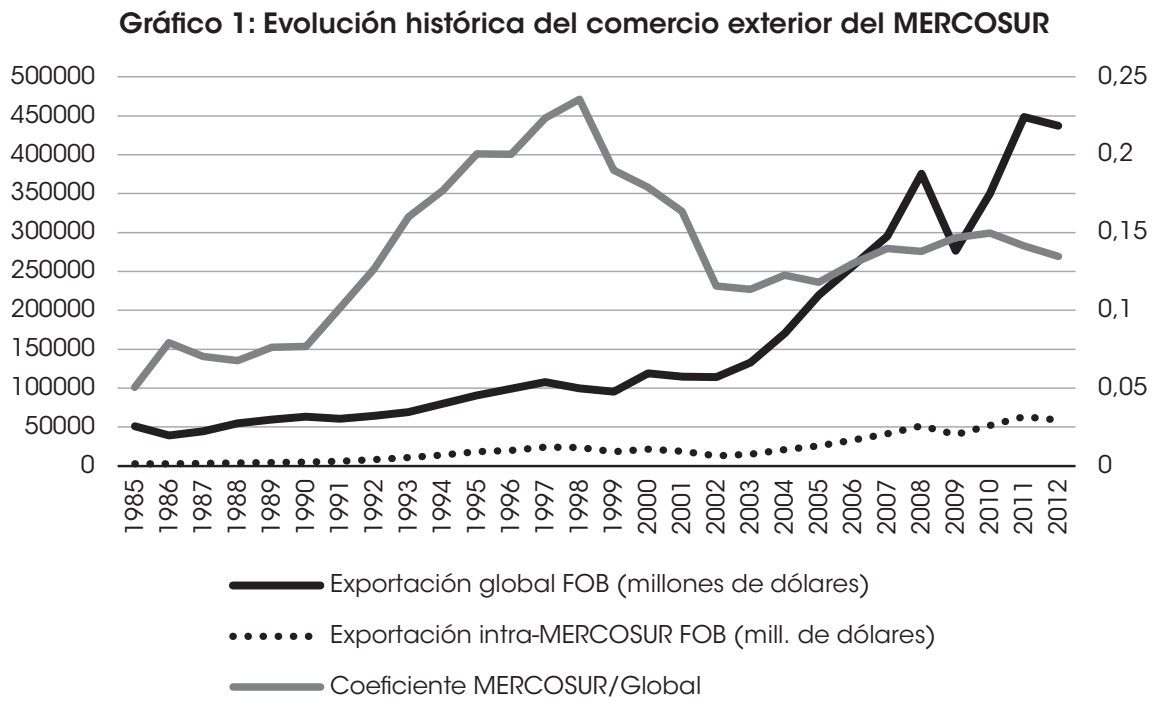

Fuente: Elaboración propia en base a datos de SICOEX-ALADI

\section{La coordinación de la política comercial de los países del MERCOSUR en la última década: una materia pendiente}

En su etapa fundacional, el MERCOSUR pretendía ser mucho más que un mercado preferencial para los países miembros. Ambicionaba alcanzar cierto piso de coordinación de políticas, y también ser una plataforma para la inserción de los países miembros en la globalización. Esta coordinación, sin embargo, no llegó a cimentarse. La aceleración que se impuso en la década del 90 fue a costa de la calidad del proceso de integración, dejando tras de sí una agenda de temas pendientes, como se ilustró en el apartado anterior. Los años subsiguientes no lograron compensar ese déficit fundacional. Si bien en la primer década

5 A diciembre de 2014, el Estado Plurinacional de Bolivia se encuentra en vías de cumplimentar su proceso de adhesión al MERCOSUR como miembro pleno. 
de los 2000 se produjo el nuevo "momento" de convergencias políticas, a raíz del "giro a la izquierda" de los ejecutivos de la región (Dabéne 2004), éste no fue suficiente para revertir esa vacancia. En consecuencia, las divergencias en materia de la política comercial externa se mantuvieron a la orden del día, especialmente a partir del año 2008, al desatarse un contexto externo menos favorable a las economías de la región.

Estas divergencias se manifestaron, en primer término, en el funcionamiento del MERCOSUR como zona de libre comercio, donde se han registrado dificultades en la coordinación de las políticas nacionales y en el cumplimiento de los acuerdos regionales. En efecto, la implementación en el plano nacional de instrumentos de administración del comercio ha condicionado la circulación de los bienes. A pesar de que el Tratado de Asunción estableció "la libre circulación de bienes, servicios y factores productivos entre los países, a través, entre otros, de la eliminación de los derechos aduaneros y restricciones no arancelarias a la circulación de mercaderías y de cualquier otra medida equivalente", el comercio entre los países del MERCOSUR ha estado afectado por instrumentos que han oficiado como restricciones de esos flujos comerciales.

En particular desde el estallido de la crisis financiera internacional, los países han recurrido a un "nuevo proteccionismo" para "evitar contagios" a partir del comercio, y preservar los mercados nacionales desde una óptica mercantilista. En estas acciones, el comercio de intrazona no ha sido exceptuado, lo que ha evidenciado una falta de coordinación de políticas intrabloque. De acuerdo al Global Trade Alert, pueden observarse entre los estados miembros del MERCOSUR la implementación de barreras no arancelarias entre sí, aun si éstas resultan contrarias a los principios de la zona de libre comercio. Se trata, entre otros, de licencias no automáticas de importación y otras equivalentes; y medidas de defensa de la competencia, tales como salvaguardias, derechos antidumping y medidas compensatorias (Gráfico 2). Estas medidas no han exceptuado a los socios regionales, sino que en su mayoría se han aplicado a las importaciones provenientes de los países más próximos (Duran Lima, Terra y Zaclicever, 2011). Por ejemplo, en los casos de las medidas antidumping, donde se produce una discriminación por origen, cabe notar que en Argentina, Brasil es el segundo mercado al cual se le aplican medidas antidumping, detrás de China (CNCE, 2013). 


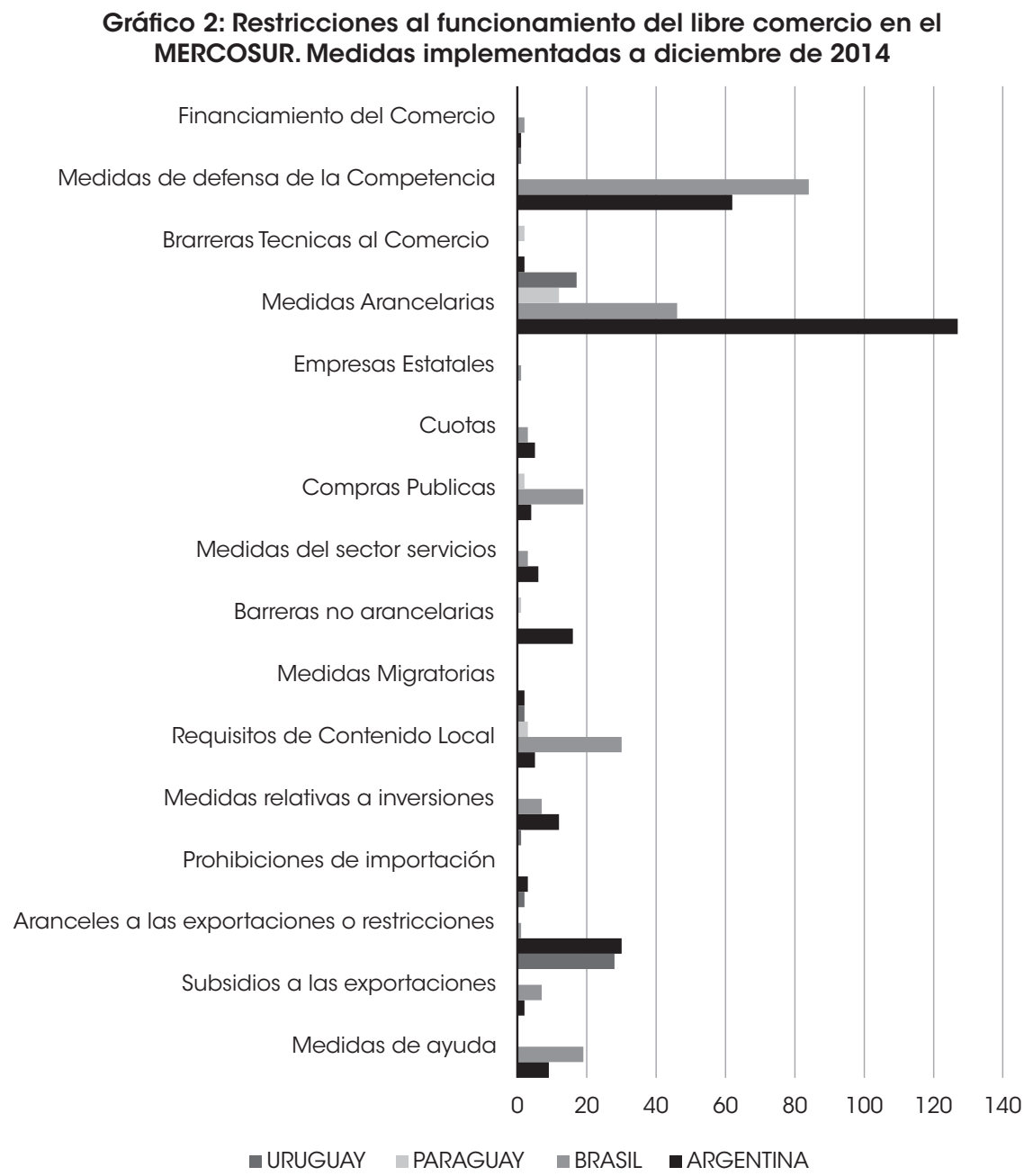

Fuente Elaboración propia en base a GTA

La implementación de estas medidas refleja un privilegio por los mercados domésticos, que ha derivado en distintas quejas, confrontaciones y reclamos entre los países del bloque; y también en críticas por parte de la sociedad civil respecto de la conveniencia del propio MERCOSUR. Argentina fue el miembro con mayor cantidad de medidas implementadas, y con mayor impacto sobre el comercio. Ello provocó que, por ejemplo, durante las reuniones bilaterales desarrolladas con Brasil en octubre de 2004, mayo y septiembre de 2005, agosto de 
2006 , febrero de 2007, octubre de 2008, febrero, marzo y noviembre de 2009, mayo y octubre de 2010, y enero y febrero 2011, se incluyeran dentro de la agenda reclamos por parte de Brasil respecto de las licencias no automáticas impuestas por la Argentina. También Uruguay y Paraguay elevaron reclamos al socio argentino. Por su parte, Argentina impuso ante Brasil una controversia ante el "Mecanismo de solución de diferencias" de la OMC por medidas antidumping sobre resinas (2006).

Las respuestas a la crisis mundial fueron, tal como caracterizó el canciller paraguayo, Héctor Lacognata, "medidas proteccionistas unilaterales, sin coordinación regional alguna y con un fuerte carácter restrictivo" (INAI, 2009). Ex post se implementaron mecanismos de consulta a nivel ministerial, por parte de Argentina, plasmados en Comités de Monitoreo Comercial con Brasil (2003), Uruguay (2006) y Paraguay (2010), que buscaron suavizar el impacto de los instrumentos implementados. Así, en vez de buscar la aplicación de los compromisos establecidos en el Tratado de Asunción y en los acuerdos subsiguientes, se privilegiaron las respuestas unilaterales y se destinaron esfuerzos diplomáticos para intentar minimizar el impacto de las trabas impuestas.

En segundo lugar, el funcionamiento del MERCOSUR como una unión aduanera también se ha visto afectado por las dificultades en la coordinación de políticas para el cumplimiento de las normas regionales. Con el paso del tiempo, los pedidos de excepciones desplazaron al funcionamiento normal acordado a partir del Protocolo de Ouro Preto. Conforme analiza Bouzas, "Los miembros no han podido arbitrar sus diferencias de intereses e incentivos y dar origen a trade offs dinámicos que sean aceptables para todas las partes. Ante esta dificultad, la condición para el mantenimiento del compromiso formal con el proyecto de unión aduanera fue, paradójicamente, su no materialización a través de excepciones negociadas y medidas ad hoc" (Bouzas, 2011: 83).

En la última década, este razonamiento puede verse reflejado en el funcionamiento de los distintos órganos y comisiones de la estructura institucional del MERCOSUR. Por ejemplo, en la Comisión de Comercio del MERCOSUR (CCM) ${ }^{6}$ se registraron más medidas de excepción que de acción positiva para la constitución del bloque. El arancel externo común, elemento constitutivo de la unión aduanera, ha sido objeto de modificaciones y perforaciones

6 La CCM fue creada por la Decisión 009/1994 del MERCOSUR. Allí quedó establecido que es el órgano encargado de velar por la aplicación de los instrumentos de política comercial común acordados para el funcionamiento de la unión aduanera, como así también de efectuar el seguimiento y revisión de los temas y materias relacionadas con la política comercial común, el comercio intra-MERCOSUR y con terceros países. 
con cada vez mayor frecuencia. Ello se ha implementado tanto a través de las llamadas "acciones puntuales en el ámbito arancelario por razones de abastecimiento", como también a través de resoluciones del Consejo de Mercado Común 7 . En el cuadro 3 puede verse el incremento de esta tendencia en los últimos años: en 2009 casi el 80\% de la labor de la Comisión de Comercio del Mercosur (CCM) se dedicó a abordar casos de excepciones al arancel externo común, mientras que entre 1999 y 2004 tales tareas eran solo el 20 o 30\% de las actividades de la CCM.

\section{Gráfico 3: Medidas de excepción al AEC 1999-2014}

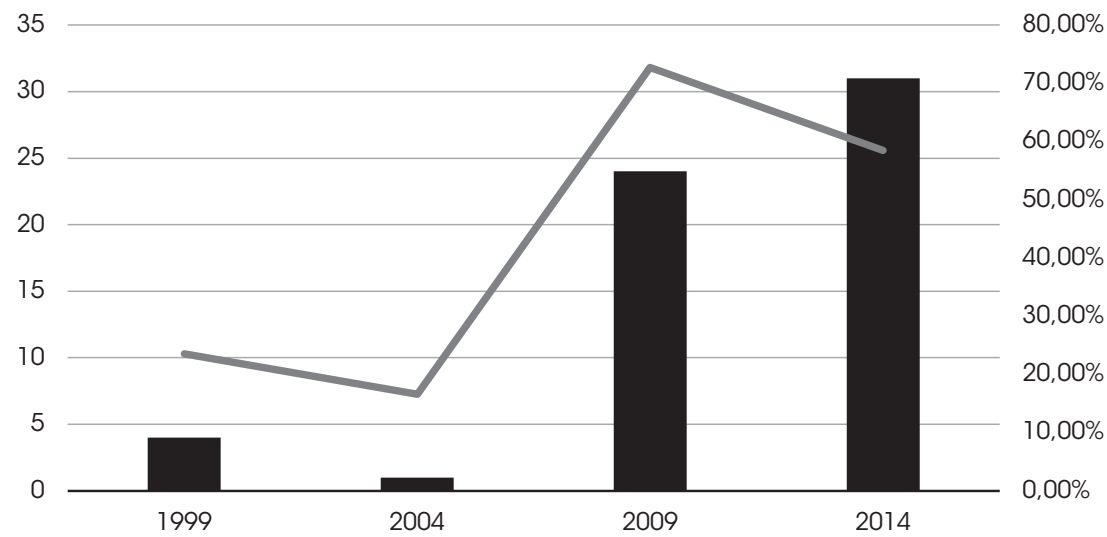

Cantidad total de medidas de excepción al AEC

Participación sobre el total de directivas del CCM

Fuente: Elaboración propia en base a datos de Consejo de Comercio de Mercosur

No solo eso, sino que, tal como evidencian Polonia Ríos y Baptista Maduro, "en 2013 se acentuó el vaciamiento de la agenda económica del MERCOSUR. En ese año, el único tema relevante tratado por el bloque fue el vinculado a las negociaciones Mercosur-UE. Además de esa negociación, la agenda interna del bloque estuvo orientada a cuestiones funcionales y administrativas" (2014:26). En los últimos años, las ampliaciones del bloque hacia nuevos socios, como Venezuela (y posteriormente Bolivia), incorporaron cronogramas de adecuación y flexibilización al arancel externo común. Ello, sumado a los pedidos de

$7 \quad$ Nos referimos, entre otras, a la Decisión CMC 39/11, que autoriza el aumento temporal del arancel externo común hasta el nivel consolidado en la Organización Mundial de Comercio a un listado de hasta 100 productos por parte de cada país, ante desequilibrios comerciales causados por la coyuntura internacional. 
excepción de los miembros orginiarios, no ha hecho más que contribuir a desdibujar la -ya difusa- union aduanera.

Como balance de estos primeros dos puntos puede afirmarse que los intercambios comerciales intrabloque del MERCOSUR no se han visto acompañados por políticas comerciales que respetasen el cumplimiento de los compromisos asumidos a lo largo de los distintos tratados y acuerdos. Menos aun se ha vislumbrado la posibilidad de avanzar hacia una integración productiva que se monte en cadenas de valor regionales ${ }^{8}$. Las políticas han sido más de "gobiernos" que de "Estado" (Barbosa, 2014); y a través de las distintas preferencias y flexibilidades implementadas puede rastrarse el peso del lobby de los sectores domésticos sensibles que, ante la falta de políticas efectivas de integración productiva o de mediación de las asimetrías, han reclamado mecanismos de protección del comercio del bloque, mayormente traducidos en excepciones a la integración regional.

Un tercer punto en el análisis de las políticas comerciales lo constituye la dimensión externa del bloque. Ésta también se ha visto afectada por las dificultades de coordinación. Tanto el Tratado de Asunción como la misma existencia de un arancel externo común, condicionan la capacidad de los países miembros para sus negociaciones comerciales externas. Sin embargo, la existencia de diferentes estrategias de inserción internacional ha llevado a flexibilizar el abordaje de la agenda externa, con pérdidas de poder relativo para el bloque, tanto en el plano multilateral como en el preferencial.

En la OMC, los países miembros del MERCOSUR actúan formalmente de manera individual y no como bloque regional. En consecuencia, su accionar conjunto ha dependido de los acuerdos intergubernamentales y de los temas abordados en la negociación, según la coyuntura específica. En términos generales, se ha producido cierta coordinación entre las diferentes delegaciones diplomáticas en Ginebra en los temas que fueran de interés para los países del bloque; así como la inclusión de las negociaciones multilaterales como área temática dentro de los organismos del MERCOSUR. Desde el año 2005 los temas de la OMC son abordados dentro del Grupo ad hoc de Consulta y Coordinación para las Negociaciones en el

8 Debe señalarse que el sector de autos y autopartes es uno de los pocos en los que se observa un desarrollo especial vinculado a la integración productiva, a partir de la existencia del Protocolo adicional al ACE 14. Sin embargo, este protocolo, de carácter provisional, no ha logrado aún ser reemplazado por un tratado de mayor jerarquía. Mientras que en 2013 no pudo acordarse una renovación del acuerdo, en 2014 Argentina y Brasil acordaron una prórroga del status-quo, y no hubo capacidad para dar respuestas comunes a una crisis que las industrias de ambos países compartían en ese momento. 
ámbito de la OMC y del SGPC (GAH OMC-SGPC) 9 . No obstante, la capacidad para labrar conductas negociadoras conjuntas en el plano multilateral ha sido acotada y ha dependido más de la voluntad política que de las capacidades institucionales del bloque.

Durante las negociaciones de la Ronda Doha, los países del MERCOSUR actuaron conjuntamente en el Grupo Cairns, y también en el G20, ambas coaliciones agrícolas. Sin embargo, no lograron niveles de coordinación equivalentes en materia de acceso a mercados no agrícolas. El momento de mayor disonancia para el bloque fue en julio de 2008, cuando en el marco de una reunión miniministerial la delegación de Brasil decidió actuar por fuera de lo acordado previamente por el MERCOSUR y aceptar la propuesta del entonces Director General, Pascal Lamy, poniendo en evidencia las limitaciones de los mecanismos de coordinación del bloque para arbitrar las diferencias en el accionar externo (Zelicovich, 2013).

En cuanto a las negociaciones preferenciales con terceros Estados, las revisiones del llamado patrimonio histórico con los socios de la ALADI se desarrollaron entre 1995 y 2004. En muchos casos se aplicaron calendarios diferenciados de reducciones arancelarias y distintas listas de excepciones para cada uno de los países miembros de manera diferenciada, bajo un único paraguas de acuerdo marco. En dicha forma, el bloque actuando conjuntamente terminó trasladando en la relación con terceros países las dificultades de sus asimetrías internas, y no demostró mayor capacidad negociadora que la de sus partes al actuar en forma individual.

En cuanto a las negociaciones extrarregionales, el MERCOSUR logró celebrar acuerdos con socios comerciales poco relevantes. En los últimos diez años firmó acuerdos de libre comercio con Israel y con el South African Custom Union (SACU), y acuerdos de preferencias arancelarias con la India. En los mismos, la lógica predominante ha sido el establecimiento de mínimos coincidentes entre los diferentes intereses nacionales, sobre una base netamente intergubernamental -por oposición a supranacional- (Zelicovich 2009:39). Otras negociaciones claves, como las de MERCOSUR con EEUU y con la Unión Europea, han presentado mayores dificultades para la coordinación externa del bloque, en tanto y en cuanto más cantidad de sectores económicos sensibles de cada uno de los Estados parte se encontraban afectados en estas negociaciones. También ha sido difícil alcanzar la coordinación interbloque en estas negociaciones, debido a los diferentes lugares que estos

9 Este grupo fue creado como un área específica del Grupo Ad Hoc de relacionamiento externo, constituido en Ouro Preto (1995) como parte del Grupo Mercado Común. 
dos socios comerciales han recibido en las estrategias de inserción internacional de cada uno de los países miembros.

En tanto que la negociación con Estados Unidos ha sido dejada de lado -puede argumentarse que ello ha estado vinculado, entre otros, al desarrollo y fracaso de la iniciativa ALCA y el posterior cambio en la política comercial externa norteamericana-, la negociación MERCOSUR-UE sigue siendo la principal negociación de la agenda externa del MERCOSUR. Las asimetrías entre las partes y las diferencias en las estrategias de inserción de cada uno de los países han dificultado el proceso negociador, y en particular la presentación y discusión de las distintas propuestas negociadoras. Las divergencias en la coordinación intrabloque han sido a su vez utilizadas por la contraparte europea para dilatar el proceso negociador en algunos momentos. Por parte del MERCOSUR, como también por parte de la Unión Europea, el proceso negociador ha estado signado por momentos de dinamismo y -muchos más- momentos de estancamiento. Tras el establecimiento del Acuerdo Marco en 1995, una primer etapa de negociaciones fue de julio 2001 a octubre 2004, cuando las diferencias entre las ofertas de las partes llevaron a un impasse; en 2007 se relanzaron las negociaciones, pero rápidamente fueron dejadas de lado por la crisis; y fueron retomadas tras la conferencia de Madrid de 2010. Los intercambios de ofertas desde entonces, no obstante, no han encontrado los consensos entonces esperados, provocando críticas de sectores empresarios de países como Brasil y Uruguay.

En los últimos meses, el tema que ha ingresado a la agenda externa ha sido el del desafío que plantea al bloque de la Alianza del Pacífico, y de manera indirecta, las negociaciones megarregionales. No se trata tanto de las preferencias arancelarias que se obtengan con la contraparte sudamericana ${ }^{10}$, sino más bien del tipo de inserción internacional que se plantea. Las miradas críticas sobre las capacidades del MERCOSUR para afrontar la globalización del siglo XXI han ido en ascenso tanto entre sectores empresarios como académicos ${ }^{11}$, a medida que las negociaciones de acuerdos megarregionales cobraban mayor dimensión y la Alianza del Pacifico ganaba reconocimiento. Las interpretaciones desde la corriente liberal sostienen que el MERCOSUR debería prontamente arribar a un acuerdo con la Unión Europea y de tal manera integrarse a una zona ampliada del Acuerdo Transatlántico (TTIP), de manera

10 Los países del MERCOSUR ya poseen acuerdos con beneficios arancelarios con los países miembros de la Alianza del Pacífico. Mantienen tratados de libre comercio con Chile (1996), Perú (2004) y Colombia (2005), mientras que con México han firmado un Acuerdo Marco (2002).

11 Por ejemplo, Polonia Ríos y Baptista Maduro (2014) plantean que "La unión aduanera del MERCOSUR se aleja a pasos agigantados de los nuevos acuerdos comerciales regionales que se van cristalizando en las diferentes regiones del mundo" 
semejante a la vinculación de la Alianza del Pacífico con el Acuerdo Transpacífico (TPP). Desde el neodesarrollismo y el estructuralismo, las valoraciones de estos acuerdos son mucho menos promisorias, y en cambio se potencia la preservación y consolidación del mercado regional. Organismos como la CEPAL, por su parte, plantean miradas mucho más optimistas sobre las posibilidades y potencialidades de "convergencia" entre la Alianza del Pacifico y MERCOSUR.

En términos generales, la agenda externa ha tenido menores éxitos de lo esperado: la coordinación ha sido coyuntural, y en los nuevos acuerdos que han podido celebrarse, el volumen de comercio abarcado es bajo. Este desempeño ha estado condicionado por el peso de las variables domésticas de cada uno de los Estados, en contraposición al interés comunitario. Coincidimos con Malamud (2013), quien sostiene que en la actualidad el "MERCOSUR sufre del síndrome de Rashomon: cumple con una función diferente para cada miembro", y ello se refleja en las orientaciones que cada uno de éstos implementa en la formulación de políticas.

\section{Los vínculos comerciales en la última década: ¿hasta dónde está lleno el vaso?}

Después de 20 años de la firma del Protocolo de Ouro Preto, difícilmente pueda afirmarse que el MERCOSUR se adapta a lo que la teoría económica establece como características de una unión aduanera. Más aun, lejos está de haber alcanzado el pretendido status de mercado común. Como se expuso en el apartado anterior, tanto en las políticas hacia la zona de libre comercio como en las relativas a la unión aduanera y a la agenda externa común, los países miembros del MERCOSUR han mantenido una cooperación limitada, que en la última década condujo en más de una ocasión a reclamos entre las partes. Aun así, desde su origen la creación del MERCOSUR ha implicado el establecimiento de crecientes flujos comerciales. En los últimos años se ha logrado cierta estabilidad, la cual puede interpretarse como un piso de consolidación.

Los procesos de integración tienen su inicio formal con la firma de acuerdos intergubernamentales, pero sólo logran transformarse en realidad cuando son incorporados a la vida de los ciudadanos y empresas de los territorios involucrados. Así, a pesar de las dificultades en la coordinación de políticas entre los gobiernos del MERCOSUR, puede apreciarse en la Argentina, Brasil, Uruguay y Paraguay una "generación" de empresas para las cuales el MERCOSUR es una parte constitutiva de su negocio. Para ello es preciso revisar tres 
indicadores: el volumen y comportamiento del comercio intrazona; el índice de intensidad del comercio; y la composición de esos flujos comerciales.

\subsection{El comercio intrazona}

Durante la última década, el comercio intrazona ha oscilado entre el 13 y el 16\% del total de las exportaciones de los países del MERCOSUR, con un valor promedio del 14\% (Cuadro 1). Se observa una expansión suave desde 2003 a 2010. Incluso en 2009, año en el que se contrajo el comercio mundial un $12.2 \%$, el MERCOSUR mantuvo los niveles previos de comercio intrazona $^{12}$. Sólo durante los años 2011 y 2012 se produjo una retracción, que fue compensada en 2013. Comparado con la década anterior (Gráfico 1), el comportamiento de los flujos del comercio intrazona mostró signos de una creciente estabilidad.

Cuadro 1

Comercio intrarregional del MERCOSUR 2004-2013

\begin{tabular}{lccccccccccc}
\hline & $\mathbf{2 0 0 4}$ & $\mathbf{2 0 0 5}$ & $\mathbf{2 0 0 6}$ & $\mathbf{2 0 0 7}$ & $\mathbf{2 0 0 8}$ & $\mathbf{2 0 0 9}$ & $\mathbf{2 0 1 0}$ & $\mathbf{2 0 1 1}$ & $\mathbf{2 0 1 2}$ & $\mathbf{2 0 1 3}$ & Promedio \\
\hline $\begin{array}{l}\text { Indice de comercio } \\
\text { intrarregional } \\
\text { (exportaciones) }\end{array}$ & $13 \%$ & $14 \%$ & $14 \%$ & $15 \%$ & $15 \%$ & $16 \%$ & $16 \%$ & $15 \%$ & $14 \%$ & $15 \%$ & $14 \%$ \\
\hline $\begin{array}{l}\text { Variación interanual } \\
\text { total }\end{array}$ & - & $0 \%$ & $1 \%$ & $1 \%$ & $0 \%$ & $0 \%$ & $0 \%$ & $0 \%$ & $-1 \%$ & $1 \%$ & $-1 \%$ \\
\hline $\begin{array}{l}\text { Variación interanual } \\
\text { porcentual }\end{array}$ & - & $2 \%$ & $4 \%$ & $6 \%$ & $2 \%$ & $1 \%$ & $1 \%$ & $-3 \%$ & $-6 \%$ & $5 \%$ & $5 \%$ \\
\hline
\end{tabular}

Elaboración propia en base a datos de la OMC: "Estadísticas del comercio internacional", 2014.

Las oscilaciones menores que han sido observadas (años 2007,2011-2012,2013) fueron explicadas como una función del nivel de actividad de las economías domésticas. Conforme a los estudios del BID-INTAL, el comercio intrazona del MERCOSUR crece en los ciclos expansivos y se contrae en épocas de crisis (BID INTAL, 2014). Así, mientras la expansión de 2007 puede adjudicarse a altas tasas de crecimiento de las economías, las contracciones de 2011-2012 se siguen del impacto de la crisis financiera internacional en las economías nacionales y de la implementación de medidas de administración del comercio; en tanto que la expansión de 2013 remite a una corrección de la contracción anterior. En su conjunto,

12 Sostiene Quijano (2011) que el MERCOSUR se constituyó en un refugio ante la crisis global que permitió que se mantuviera el nivel de actividad, frente a la caída de la participación relativa de las exportaciones industriales hacia el resto del mundo. 
estas oscilaciones han sido menores y puede ponderarse el período como uno de relativa estabilidad en la historia del bloque regional.

Esta evolución, sin embargo, no ha sido homogénea entre los miembros del bloque. Al desagregar entre los cuatro miembros originarios del MERCOSUR se observa que, para cada uno de ellos, el comercio intrazona ha tenido una participación diferente, en función de las asimetrías que existen de manera estructural. Como constante a lo largo de la década, el bloque ha sido, en términos comerciales, mucho más importante para Argentina, Uruguay y Paraguay que para Brasil. Por su parte, puede observarse que Uruguay ha diversificado los destinos de sus exportaciones, siendo en 2013 el MERCOSUR menos importante como destino de sus exportaciones de lo que era en 2003. En el caso de Argentina ha ocurrido la tendencia opuesta, en tanto que Paraguay observa importantes fluctuaciones, principalmente debido al peso de la energía eléctrica en el total de sus exportaciones (Gráfico 4).

Estas asimetrías generan distintos efectos en términos de la sensibilidad y vulnerabilidad de las políticas nacionales a los cambios externos. Conforme a la teoría de la interdependencia compleja, la "sensibilidad implica grados de respuesta dentro de una estructura política ( ¿con qué rapidez los cambios en un país ocasionan cambios, con determinado costo, en otro país y cuál es la magnitud de ese costo?)", mientras que la vulnerabilidad “puede definirse como la desventaja de un actor que continua experimentando costos impuesto por acontecimientos externos aun después de haber modificado las políticas" (Keohane y Nye, 1988). El comercio intrazona genera lazos de sensibilidad en las relaciones de interdependencia entre los miembros del MERCOSUR; sin embargo, a raíz de las asimetrías en la distribución de ese comercio intrazona, Brasil resulta mucho menos vulnerable a los cambios externos que los demás miembros del bloque regional. Este argumento, que se remonta a rasgos que son estructurales del MERCOSUR, puede explicar en parte algunas de las divergencias en materia de cooperación en las relaciones comerciales internacionales de la última década señaladas más arriba, por ejemplo, en cuanto al accionar diferente de Brasil en las negociaciones de la Organización Mundial de Comercio. 


\section{Gráfico 4: Participación en el comercio intrazona}

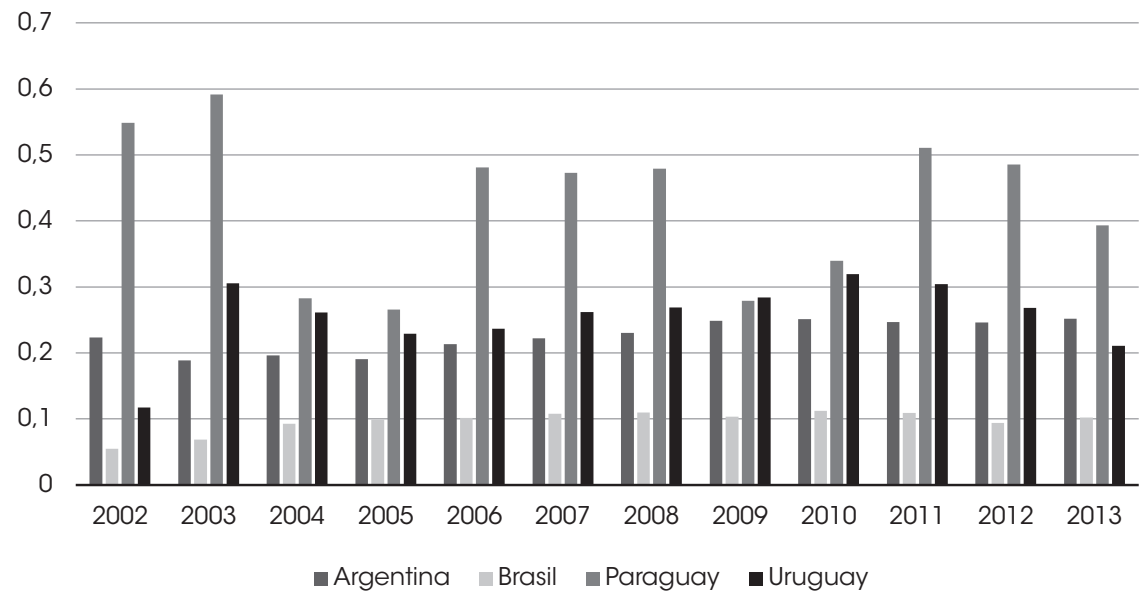

Fuente: Elaboración propia en base a datos de SICOEX ALADI

En resumen, el comercio intrazona ha mantenido en la última década un comportamiento relativamente estable. La participación de cada uno de los miembros en este comercio ha continuado siendo asimétrica, con mayor grado de integración al bloque por parte de las economías más pequeñas que de Brasil. La estabilidad de los intercambios sostenida a lo largo de la década permite prever que se trata de un piso de funcionamiento del MERCOSUR que podría sostenerse en el mediano plazo, incluso a pesar de que estos niveles se encuentren debajo del potencial ${ }^{13}$ del bloque, en función de sus indicadores historicos.

\subsection{El índice de intensidad del comercio}

Otros procesos de integración, como el NAFTA o la UE, presentan niveles de comercio intrarregional mucho mayores que el 14\% del MERCOSUR ${ }^{14}$. Ello ha sido un argumento frecuentemente utilizado por los críticos del bloque regional. Sin embargo, estas críticas omiten el hecho de que una de las razones por la cual este indicador es más bajo que en

13 El índice del comercio intrarregional potencial es "una medida del mayor o menor grado de aprovechamiento de las preferencias intrarregionales que los países se otorgan recíprocamente dentro de una agrupación regional" (Durán Lima y Álvarez, 2011 :90), en función de los registros históricos del comercio de los países de la agrupación. En Durán Lima y Lo Turco (2010) se constata que, dentro de la ALADI, para el año 2008 tanto el MERCOSUR como la CAN se encontraban debajo de su comercio intrarregional potencial.

14 El NAFTA y la UE tienen valores promedio de $49 \%$ y $59 \%$, respectivamente (OMC, 2013) 
otras economías es que las economías que integran el MERCOSUR son relativamente más pequeñas y presentan menores niveles de apertura.

El Índice de Intensidad del Comercio (IIC) (Durán Lima y Álvarez, 2011) permite corregir esa distorsión en la medición al ponderar el comercio intrazona con la relevancia del bloque en el comercio mundial. Cuando el valor del índice es mayor (o menor) a la unidad, se constata la existencia de un sesgo geográfico en los intercambios comerciales, en tanto que si el valor es de 1, ello indica una neutralidad o equilibrio. Por su parte, un cambio positivo indica que el comercio entre los socios creció más rápido de lo que cabría esperar en base al cambio en la participación del socio en el comercio mundial (neto de las importaciones del país), y viceversa.

\section{Cuadro 2}

Índice de Intensidad del Comercio del MERCOSUR 2004-2013

\begin{tabular}{lccccccccccc}
\hline & $\mathbf{2 0 0 4}$ & $\mathbf{2 0 0 5}$ & $\mathbf{2 0 0 6}$ & $\mathbf{2 0 0 7}$ & $\mathbf{2 0 0 8}$ & $\mathbf{2 0 0 9}$ & $\mathbf{2 0 1 0}$ & $\mathbf{2 0 1 1}$ & $\mathbf{2 0 1 2}$ & $\mathbf{2 0 1 3}$ & Promedio \\
\hline $\begin{array}{l}\text { Indice de } \\
\text { intensidad del } \\
\text { comercio }\end{array}$ & 9.04 & 8.67 & 9.00 & 9.38 & 8.85 & 8.89 & 8.50 & 7.85 & 7.78 & 8.27 & 8.66 \\
\hline $\begin{array}{l}\text { Variación } \\
\text { interanual total }\end{array}$ & - & -0.37 & 0.33 & 0.37 & -0.53 & 0.04 & -0.39 & -0.65 & -0.07 & 0.50 & 0.38 \\
\hline $\begin{array}{l}\text { Variación } \\
\text { interanual } \\
\text { porcentual }\end{array}$ & - & $-4 \%$ & $4 \%$ & $4 \%$ & $-6 \%$ & $0 \%$ & $-4 \%$ & $-8 \%$ & $-1 \%$ & $6 \%$ & $6 \%$ \\
\hline
\end{tabular}

Elaboración propia en base a datos OMC. "Estadisticas del Comercio Internacional", 2014

En el caso del MERCOSUR, observamos en la década 2003-2013 valores relativamente elevados del IIC, con un 8.66 promedio. Es decir que las exportaciones de los miembros del MERCOSUR a sus socios intrazona son más relevantes de lo que tales socios son en el mundo. Debe llamarse la atención que, al considerar las variaciones interanuales, lo que surge es que, especialmente desde 2008, la leve baja en el comercio intrazona observada en el apartado anterior (Cuadro 1) ha sido acompañada por una disminución mayor en el Índice de Intensidad del Comercio (Cuadro 2). Esto es, no solo hay una contracción del comercio por un contexto recesivo, sino también una preferencia hacia socios comerciales de extrazona, lo que conlleva una menor intensidad del comercio entre los socios del MERCOSUR. Por ejemplo, desde 2008 en adelante se observa una creciente participación de China como destino de las exportaciones de la región (Cuadro 2), lo que podría indicar una correlación entre la disminución del IIC del MERCOSUR y la presencia del socio asiático. 


\section{Cuadro 3 \\ Participación de las exportaciones a China sobre el total de exportaciones del MERCOSUR}

\begin{tabular}{lcccccc}
\hline & $\mathbf{2 0 0 8}$ & $\mathbf{2 0 0 9}$ & $\mathbf{2 0 1 0}$ & $\mathbf{2 0 1 1}$ & $\mathbf{2 0 1 2}$ & $\mathbf{2 0 1 3}$ \\
\hline $\begin{array}{l}\text { Exportaciones a China / } \\
\text { exportaciones totales del MERCOSUR }\end{array}$ & $8 \%$ & $11 \%$ & $13 \%$ & $14 \%$ & $14 \%$ & $16 \%$ \\
\hline $\begin{array}{l}\text { Intensidad del comercio de } \\
\text { MERCOSUR a China }\end{array}$ & 0.93 & 1.16 & 1.28 & 1.38 & 1.24 & 1.33 \\
\hline
\end{tabular}

Fuente: Elaboración propia en base a datos de COMTRADE

Si se compara con décadas anteriores, el IIC entre los años 2003 y 2013 exhibe valores más bajos, pero más estables que en la década del 90 , donde hubo un importante crecimiento seguido por una caída más acentuada en el periodo 1998-2002 (Durán Lima y Álvarez, 2011). El final del período estudiado muestra mayores niveles de comercio intrazona, pero menor intensidad del comercio.

\subsection{La composición del comercio}

El tercer elemento que debe incorporarse al análisis de las relaciones comerciales del MERCOSUR es el de la composición del comercio. Los intercambios intrabloque se destacan por presentar una mayor agregación de valor que el resto de las exportaciones de las mismas economías ${ }^{15}$. No obstante, salvo pocas excepciones, en el comercio de manufacturas de origen industrial predominan los bienes finales por sobre los insumos, lo que indicaría bajos niveles de integración productiva.

Un recuento de los primeros 10 productos comercializados por cada una de las partes hacia la intrazona ${ }^{16}$ muestra que el comercio de Argentina y Brasil hacia la región se concentra en autos y autopartes -tanto importaciones como exportaciones-; Uruguay concentra los primeros 10 productos de importación en dos sectores: vehículos y productos de la cadena alimenticia. Exporta alimentos y productos primarios; vehículos y manufacturas de las industrias del caucho (planchas), química (fungicidas) y hierro (tubos). El principal producto de exportación de Paraguay al resto de la intrazona es la energía eléctrica (60\%), mientras

15 Conforme a datos de la ALADI, para el año 2013, las manufacturas representaban el $72.7 \%$ de las ventas de Argentina al MERCOSUR, pero solo el $33.1 \%$ de las exportaciones de este país al mundo. Para Brasil, los valores son 83.3\% en MERCOSUR y $35.9 \%$ en las exportaciones totales. En Uruguay, las manufacturas son $56.9 \%$ de las ventas al MERCOSUR, pero $23.2 \%$ en las totales.Y por último, en el caso de Paraguay, se constata un $8.08 \%$ en el MERCOSUR y $11.5 \%$ en las totales.

16 Fuente: SICOEX ALADI 
que los 9 restantes son todos productos primarios vinculados a la cadena alimenticia. Sus importaciones son combustibles, vehículos, cereales y tabaco.

Las cadenas de valor intrarregionales son aún limitadas, siendo éste uno de los temas pendientes más relevantes del MERCOSUR. Se destaca no obstante la del sector automotriz, que por ejemplo concentró más del 50\% del comercio bilateral de Argentina y Brasil en 2014. De manera incipiente, Uruguay también ha orientado su producción hacia la elaboración de insumos para otras industrias de la región, incluida la del sector automotriz.

La agregación de valor de las exportaciones ha crecido en la última década (Cuadro 8). En el caso de Argentina, debe señalarse la creciente participación de los vehículos y autopartes entre los principales productos exportados en 2013, vis a vis, los del año 2003. En el caso de Brasil, también se evidencia una mayor integración de las ventas de vehículos, así como de otras manufacturas de origen industrial (MOI). Asimismo, son un capítulo creciente los combustibles. Para Paraguay, el gran cambio ha estado dado por el incremento en la exportación de energía eléctrica - destinada a la Argentina. Uruguay mantuvo constantes las manufacturas de origen agropecuario (MOA) -lácteos-, pero incrementó sus exportaciones regionales de insumos químicos e industriales, y también vehículos, como se señaló anteriormente.

\section{Cuadro 4}

\section{Composición del comercio intrazona del MERCOSUR. Porcentaje sobre el total de exportaciones al MERCOSUR (años 2003 y 2013)}

\begin{tabular}{|c|c|c|c|c|c|c|c|c|}
\hline AÑo 2003 & $\begin{array}{l}\text { Trigo y } \\
\text { otros } \\
\text { cereales }\end{array}$ & $\begin{array}{l}\text { Otras } \\
\text { MOA }\end{array}$ & $\begin{array}{l}\text { Aceites deriva- } \\
\text { dos del petróleo } \\
\text { y otros produc- } \\
\text { tos químicos }\end{array}$ & $\begin{array}{l}\text { Gas oil } \\
\text { y otros } \\
\text { combus- } \\
\text { tibles }\end{array}$ & $\begin{array}{l}\text { Otras MOI } \\
\text { - Insumos } \\
\text { para la } \\
\text { industria }\end{array}$ & $\begin{array}{l}\text { Vehículos y } \\
\text { autopartes }\end{array}$ & $\begin{array}{c}\text { Otros } \\
\text { bienes de } \\
\text { consumo } \\
\text { final }\end{array}$ & $\begin{array}{l}\text { Energía } \\
\text { eléctrica }\end{array}$ \\
\hline Argentina & $15 \%$ & $0 \%$ & $10 \%$ & $8 \%$ & $0 \%$ & $5 \%$ & $0 \%$ & $0 \%$ \\
\hline Brasil & $0 \%$ & $0 \%$ & $3 \%$ & $0 \%$ & $2 \%$ & $13 \%$ & $2 \%$ & $0 \%$ \\
\hline Paraguay & $56 \%$ & $29 \%$ & $0 \%$ & $0 \%$ & $1 \%$ & $0 \%$ & $0 \%$ & $0 \%$ \\
\hline Uruguay & $29 \%$ & $4 \%$ & $3 \%$ & $0 \%$ & $8 \%$ & $0 \%$ & $0 \%$ & $0 \%$ \\
\hline AÑO 2013 & $\begin{array}{l}\text { Trigo y } \\
\text { otros } \\
\text { cereales }\end{array}$ & $\begin{array}{l}\text { Otras } \\
\text { MOA }\end{array}$ & $\begin{array}{l}\text { Aceites deriva- } \\
\text { dos del petróleo } \\
\text { y otros produc- } \\
\text { tos químicos }\end{array}$ & $\begin{array}{l}\text { Gas oil } \\
\text { y otros } \\
\text { combus- } \\
\text { tibles }\end{array}$ & $\begin{array}{l}\text { Otras MOI } \\
\text { - Insumos } \\
\text { para la } \\
\text { industria }\end{array}$ & $\begin{array}{l}\text { Vehículos y } \\
\text { autopartes }\end{array}$ & $\begin{array}{c}\text { Otros } \\
\text { bienes de } \\
\text { consumo } \\
\text { final }\end{array}$ & $\begin{array}{l}\text { Energía } \\
\text { eléctrica }\end{array}$ \\
\hline Argentina & $3 \%$ & $0 \%$ & $4 \%$ & $0 \%$ & $0 \%$ & $36 \%$ & $2 \%$ & $0 \%$ \\
\hline Brasil & $0 \%$ & $0 \%$ & $0 \%$ & $2 \%$ & $4 \%$ & $28 \%$ & $0 \%$ & $0 \%$ \\
\hline Paraguay & $15 \%$ & $7 \%$ & $0 \%$ & $0 \%$ & $1 \%$ & $0 \%$ & $0 \%$ & $60 \%$ \\
\hline Uruguay & $15 \%$ & $4 \%$ & $3 \%$ & $0 \%$ & $13 \%$ & $8 \%$ & $0 \%$ & $0 \%$ \\
\hline
\end{tabular}




\section{Conclusiones}

A 20 años del Protocolo de Ouro Preto, la dimensión comercial del MERCOSUR transita una etapa crítica. Como hemos visto, existen dificultades en la coordinación intrabloque, tanto de las políticas constitutivas de la zona de libre comercio como de la unión aduanera y de su agenda externa. Sin embargo, los flujos del comercio intrarregionales subsisten y se mantienen relativamente estables a pesar de esos desajustes en la coordinación de políticas. De tal forma, como afirmamos a comienzos de este artículo, es preciso reconocer el nivel de interdependencia alcanzado entre los países miembros del MERCOSUR en los últimos tiempos en cuanto a sus relaciones de comercio intrazona. Puede argumentarse incluso que esta interdependencia sea la que haya conducido a que pueda observarse en el análisis una mayor autonomía entre la evolución de los flujos de comercio y la evolución de la coordinación de políticas que la esperada conforme a las interpretaciones predominantes en el área.

A modo de balance, es preciso señalar que los aspectos más positivos del MERCOSUR en cuanto a los intercambios de bienes están dados por el mantenimiento a lo largo de la última década del comercio intrazona, especialmente cuando éste es considerado en relación al peso del bloque regional en el comercio mundial; también es un elemento positivo el crecimiento de la agregación de valor en los bienes objeto del comercio. Por su parte, la persistencia de las asimetrías en la relevancia del bloque para los países miembros, la tendencia del IIC -a la baja-y la preeminencia del intercambio de bienes finales por sobre el intercambio de insumos, indican en cambio un escenario menos optimista respecto de la salud de la dimensión comercial del bloque.

Puede afirmarse entonces que, si bien el MERCOSUR no ha alcanzado los objetivos propuestos, sí ha llegado a establecer, en la última década, un nivel de comercio mínimo estable que indica la existencia de una interdependencia de algunos sectores económicos entre las partes, que difícilmente pueda ser revertida. La unión aduanera sigue siendo una deuda pendiente, difícil de alcanzar, pero el "vaso puede estar medio lleno".

Fecha de recepción: 10 de agosto de 2015 Fecha de aceptación: 12 de octubre de 2015 


\section{Referencias}

1. Alfonsín, Raúl (1987). Discursos presidenciales ("Discurso del señor Presidente de la Nación, Doctor Raúl R. Alfonsín, ante la asamblea legislativa de la República Oriental del Uruguay, el día 25 de mayo de 1987"). Buenos Aires: Subsecretaría de Comunicación Social. Dirección General de Difusión.

2. Barbosa, Rubens (2014). "El futuro del MERCOSUR." Boletín Informativo Techint, Buenos Aires, No 345.

3. BID-INTAL (2014). "Informe MERCOSUR No 19 (2013-2014) Segundo semestre 2013-Primer semestre 2014." Publicaciones del Banco Interamericano de Desarrollo. Nota Técnica IDB-TN 719. Disponible en http://publications.iadb.org/ handle/11319/6704?locale-attribute=es. Consultado en noviembre 2014.

4. Bouzas, Roberto y Emilio Pagnota (2003). Dilemas de la política comercial externa argentina. Buenos Aires: Ed. Siglo XXI.

5. Bouzas, Roberto (2011). "Apuntes sobre el estado de la integración regional en América Latina.” En: Gerardo Caetano (coord.): MERCOSUR 20 años. Montevideo: Centro de Formación para la Integración Regional CEFIR.

6. Bouzas, Roberto, Pedro Motta Veiga y Sandra Ríos (2007). “Crisis y perspectivas de la integración sudamericana”. Foreign Affairs (en español), octubre-diciembre, 2007.

7. Caetano, Gerardo (2011). "Breve historia del MERCOSUR en sus 20 años. Coyunturas e instituciones (1991-2011)”. En: Gerardo Caetano (coord.): MERCOSUR 20 años. Montevideo: Centro de Formación para la Integración Regional CEFIR.

8. Cienfuegos, Manuel y José Antonio Sanahuja (2010). Una región en construcción. UNASUR y la integración en América del Sur. Barcelona: Fundación CIDOB.

9. Comisión Nacional de Comercio Exterior, CNCE (2013). "Informe anual 2013. Comisión Nacional de Comercio Exterior”. Buenos Aires. Disponible en http://www. cnce.govar/Publicaciones/ia/ia2013/pdf/ia2013.pdf Consultado en abril de 2015.

10. Dabene, Oliver (2004). "La reactivación del MERCOSUR: Ouro Preto II o el tiempo de las reformas políticas”. Oasis, № 10. Universidad Externado de Colombia., Bogotá.

11. Duran Lima, José y Mariano Álvarez (2011). Manual de comercio exterior y política comercial. Nociones básicas, clasificaciones e indicadores de posición y dinamismo. Santiago de Chile: CEPAL-ONU. 
12. Duran Lima, José y Alessia Lo Turco (2010). "El comercio intrarregional en América Latina: patrón de especialización y potencial exportador." En: María Inés Terra y José Duran Lima: Los impactos de la crisis internacional en América Latina. ¿Hay margen para el diseño de políticas regionales? Montevideo: Red Mercosur.

13. Duran Lima, José, María Inés Terra y Dayna Zaclicever (2011). "Efectos de las medidas de política comercial aplicadas en América Latina sobre el comercio regional y extrarregional." CEPAL, Serie Comercio Internacional, No 109, Santiago de Chile.

14. Flores, Maria Cándida (2004). "De Iguaçu a Ouro Preto: as diferentes orientações do Mercosul nos discursos do governo brasileiro.” Comunicação y política, n.s., X (3):77-167.

15. Fundación Instituto para las Negociaciones Agrícolas Internacionales, INAI (2009). Boletin de Noticias No 86. Buenos Aires. Disponible en http://www.inai.org.ar/ archivos/boletines/Nuevo\%20Bolet\%C3\%ADn\%2086.pdf. Consultado en: febrero de 2015

16. Hilliar, Rubén (comp.) (1996). MERCOSUR: estado actual e historia reciente. Serie Documentos de apoyo a la capacitación. Argentina. Disponible en http:/ / www.sgp.gob. $\mathrm{ar} /$ contenidos/inap/publicaciones/docs/capacitacion/mercosu2.pdf. Consultado en febrero de 2015.

17. Keohane, Robert y Joseph Nye (1988). Poder e interdependencia: la política mundial en transición. Buenos Aires: Grupo Editor Latinoamericano.

18. Malamud, Andrés (2013). "El MERCOSUR: misión cumplida”. Revista SAAP, 7(2), Ciudad Autónoma de Buenos Aires.

19. Organización Mundial de Comercio (2013). "Informe sobre el comercio mundial." Ginebra: OMC.

20. Polonia Ríos, Sandra y Lucía Baptista Maduro (2014). "MERCOSUR: ¿llegó el momento de replantear el modelo?" Boletín informativo Techint, No 345, Buenos Aires.

21. Quijano, José Manuel (2011). “El MERCOSUR 20 años después.” En: Gerardo Caetano (coord.): MERCOSUR 20 años. Montevideo: Centro de Formación para la Integración Regional CEFIR.

22. Rapoport, Mario (2010). Las politicas económicas de la Argentina. Una breve historia. Buenos Aires: Booket.

23. Serbin, Andrés, Laneydi Martínez, Haroldo Ramanzini Júnior (2012). El regionalismo "post-liberal" en América Latina y el Caribe: nuevos actores, nuevos temas, nuevos desafíos. 
Anuario de la Integración Regional de América Latina y el Gran Caribe. Buenos Aires: Coordinadora Regional de Investigaciones Económicas y Sociales (CRIES).

24. Zelicovich,Julieta (2009). "La agenda externa del MERCOSUR: evolución, aprendizaje y expansión de las negociaciones comerciales conjuntas". Ponencia presentada en las I Jornadas de Relaciones Internacionales del Área de Relaciones Internacionales de la Facultad Latinoamericana de Ciencias Sociales (FLACSO). Buenos Aires, septiembre 2009.

25. ------- (2013). "Argentina en las negociaciones comerciales multilaterales de la Ronda Doha (2001-2011). Análisis de su participación en el proceso negociador”. Tesis presentada para la obtención del Doctorado en Relaciones Internacionales. Universidad Nacional de Rosario, Argentina. 